Revista

\title{
Multi-Ensayos
}

Vol. 7, núm. 14

ISSN: 2412-3285

https://multiensayos.unan.edu.ni

DOI: https://doi.org/10.5377/multiensayos.v7i14.12000

\section{Enseñanza y aprendizaje de la Física y Matemática Superior en Tiempos de Pandemia}

\section{Teaching and Learning of Higher Physics and Mathematics in Times of Pandemic}

\author{
Cliffor Jerry Herrera Castrillo ${ }^{1}$ \\ Donald Ariel Hernández Muñoz ${ }^{2}$
}

Recibido: 02 de abril de 2021. Aceptado: 14 de junio de 2021

\section{RESUMEN}

El presente ensayo crítico, aborda el ambiente que se vive en la educación superior, debido a los estragos provocados por la pandemia que ha paralizado al mundo COVID-19, debido a esto la manera de facilitar aprendizajes se ha tenido que redefinir en todas las áreas, y de forma particular en física y matemáticas, ya que ambas ciencias son necesarias para formar integralmente al hombre moderno, permitiéndole analizar y comprender el uso y significado de los números, realizar comparaciones de cantidades física y sistematizar la información. Es por ello que este ensayo fue realizado con el propósito de analizar desde ambientes semipresenciales la forma como se enseña y aprende física y matemática en la UNAN MANAGUA FAREM Estelí, durante primer semestre 2020.

Palabras claves: Enseñanza; aprendizaje; COVID-19; TIC; matemática; física.

\begin{abstract}
This critical essay addresses the environment that exists in higher education, due to the ravages caused by the pandemic that has paralyzed the world COVID-19, due to this the way of facilitating learning has had to be redefined in all areas, and particularly in physics and mathematics, since both sciences are necessary to comprehensively train modern man, allowing him to analyze and understand the use and meaning of numbers, make comparisons of physical quantities and systematize information. That is why this essay was carried out with the purpose of analyzing from blended environments the way how physics and mathematics are taught and learned at UNAN MANAGUA FAREM Estelí, during the first semester 2020.
\end{abstract}

Keywords: Teaching; learning; COVID-19; ICT; math; physics.

1 Docente UNAN-Managua/FAREM-Estelí. Correo electrónico: clifforjerryherreracastrillo@gmail.com

2 Docente UNAN-Managua/FAREM-Estelí. Correo electrónico: hdonaldariel@gmail.com

(c) 2021 Revista Multi-Ensayos. 


\section{INTRODUCCIÓN}

En el mundo entero, los efectos de la pandemia del COVID-19 ${ }^{1}$ han alcanzado a la educación superior, que se ha visto obligada a tomar medidas sanitarias y de distanciamiento humano para disminuir la velocidad de contagio de la pandemia y de esa forma darle protección a la población en general. El sector académico ha sido directamente afectado y forzado a tener que realizar cambios significativos en su forma de enseñar, y buscar alternativas para que los estudiantes no pierdan sus estudios universitarios. En este caso particular, es de interés analizar las experiencias en el proceso de la enseñanza - aprendizaje en el área de Física y Matemática, durante el primer semestre 2020, en UNAN MANAGUA²-FAREM Estelí3.

Debido a la crisis producida por el COVID-19, los niveles educativos, docentes, estudiantes, familias nicaragüenses y responsables académicos, de manera urgente han tratado de responder a esta situación. Por ello desde la UNAN MANAGUA, se decidió tomar las medidas de prevención como lo son el distanciamiento social, por ello los grupos de clase con más de 30 estudiantes se dividieron en 2 (Grupo A y B) , así como el uso de mascarilla obligatorio en todas las instalaciones universitarias, lavado de manos entre otras orientaciones dadas por el ministerio de salud.

¿Cómo aprenden los estudiantes las diferentes temáticas de Física y Matemática en el contexto de pandemia de COVID-19?

La pregunta antes mencionada, permite abordar en el presente ensayo, la práctica pedagógica, que se ha tenido que implementar en las asignaturas de física y de matemática, para lograr facilitar aprendizajes, científicos y prácticos, que sean de utilidad para los estudiantes, sin obviar el contexto nicaragüense, que ha sido diferente en comparación con otros países, debido a su sistema de salud pública. Donde el número de contagiados es muy bajo, esto ha permitido el desarrollo de clases presenciales acompañadas de herramientas tecnológicas, como plataformas de intercambio de información, clases a través de videoconferencia (Zoom, Meeting, Salas de Messenger), Pizarrones digitales interactivos, Cuestionarios y aplicaciones que permiten la resolución de ejercicios (Wolfram Alpha ${ }^{4}$, Scientific Workplace ${ }^{5}$, PhotoMath ${ }^{6}$, entre otras)

\footnotetext{
1 La enfermedad por coronavirus de 2019, más conocida como COVID-19 es una enfermedad infecciosa causada por el SARSCoV-2. Produce síntomas similares a los de la gripe, entre los que se incluyen fiebre, tos, disnea (dificultad respiratoria), mialgia (dolor muscular) y fatiga. En casos graves se caracteriza por producir neumonía, síndrome de dificultad respiratoria aguda, sepsis y choque séptico que conduce a cerca de 3,75 \% de los infectados a la muerte según la OMS. No existe tratamiento específico; las medidas terapéuticas principales consisten en aliviar los síntomas y mantener las funciones vitales

2 Universidad Nacional Autónoma de Nicaragua UNAN MANAGUA

3 Facultad Regional Multidisciplinaria de Estelí

4 Wolfram Alpha es un buscador online que responde a preguntas y realiza cálculos de manera inmediata. Sus respuestas son detalladas y específicas a los conceptos introducidos en su motor de búsqueda en lugar de proporcionar una lista de documentos o páginas web como hacen otro tipo de buscadores

5 Scientific WorkPlace es un procesador de texto para crear y ver documentos LaTeX. Scientific WorkPlace le permite ingresar notación matemática seleccionando símbolos de paletas o barras de herramientas.

6 PhotoMath es una aplicación móvil descrita como una calculadora por cámara, que utiliza la cámara del teléfono móvil para reconocer patrones matemáticos y mostrar la solución directamente en la pantalla. Es gratuita y está disponible para Google Android e iOS.
} 
Herrera (2021) plantea que:

Dentro de los medios de comunicación, que todos los estudiantes tienen acceso, están las redes sociales ya que de una u otra forma todos poseen, aunque sea una (Facebook, Messenger, WhatsApp, Instagram, entre otras), por lo que resulta pertinente trabajar mediante estos medios, además de que resultan más económicos para los estudiantes, un ejemplo de ello son las salas de Messenger, que consumen menos datos que utilizar Zoom, Meeting u otro recurso para video conferencias. (p. 16)

En el pasado, la mayoría de las instituciones de educación superior han actuado con lentitud en aprovechar el potencial de las nuevas tecnologías. Si se hubiese tenido una mayor apertura y flexibilidad al respecto, tal vez la transición hubiese sido menos disruptiva. En el futuro, la educación superior seguramente no será la misma que prevalecía antes de la pandemia, ya que las opciones no presenciales se convertirán en una alternativa más usada a partir de ahora. (Sánchez, 2020). De acuerdo a lo antes mencionado, en este ensayo se muestran elementos que llevan a la reflexión de como el aprendizaje de la Física y de Matemática en la era digital, en carreras de ingenierías, ciencias económicas y de la educación, donde es de importancia, para facilitar conocimientos, mediante la comunicación e información, que ayuden a cumplir objetivos propuestos.

El COVID-19 ha mostrado que el sector educativo necesita cambios trascendentales en la forma como los docentes deben sobrellevar los nuevos escenarios que han surgido en consecuencia directa de una pandemia que provoca deserción de estudiantes, dolor e incertidumbre en la educación.

\section{DESARROLLO}

La educación es un proceso fundamental para el desarrollo de las capacidades humanas, y lograr gozar de una vida plena, la presencia y participación de una ciudadanía activa. Por ello, forma parte de los derechos humanos universales, clave para la convivencia. Garantizar este derecho es responsabilidad de todas y todos, pero en primer lugar del Estado; por ello, la Constitución nicaragüense lo establece en los artículos 58, 116, 117 y 121, para hacerlo efectivo.

Es por ello, que en Nicaragua respetando el derecho a la educación de todos los ciudadanos el Consejo Nacional de Universidades CNU, (2020) plantea:

... "Seguir promoviendo las medidas de prevención contenidas en el protocolo de salud de nuestro país, con la confianza en todos los pasos que se han venido desarrollando que demuestran el sentido de la responsabilidad de nuestras autoridades nacionales y de los integrantes de la comunidad universitaria; continuaremos desarrollando todas nuestras acciones con calma, serenidad y confianza." (p.1)

En el contexto de Nicaragua, desde 2018 ya se vienen utilizando las tecnologías en el aula, debido a situaciones sociopolíticas ${ }^{7}$ que vivía el país en ese entonces, pero gracias al gobierno fueron solucionadas.

7 Las protestas de 2018 en Nicaragua fueron una serie de manifestaciones que se iniciaron el 18 de abril de 2018 por las reformas al sistema de seguro social, en donde pequeños grupos manipulados por personas opositoras al gobierno, no permitieron la libre circulación en el país, por lo que las universidades optaron por clases en línea, estos tranques gracias a la buena voluntad del gobierno y el apoyo de la policía Nacional, se logro regular desapareciendo los denominados "tranques" 
La tecnología estimula el aprendizaje y se puede dar lugar a métodos pedagógicos más efectivos donde se obtienen excelentes resultados. Los estudios realizados en el mundo en la última década ya advertían esta realidad, pero ha tenido que llegar una pandemia para que muchos docentes logren ver la utilidad e importancia de las Tecnologías de la Información y la Comunicación (TIC) en las diferentes asignaturas, principalmente las relacionadas a física y matemática, que requieren de un tratamiento analítico y reflexivo para lograr ser comprendidos.

En la Facultad Regional Multidisciplinaria de Estelí (FAREM - Estelí), universidad ubicada al norte de Nicaragua, en total son ocho carreras que durante toda su duración reciben y desarrollan asignaturas de física y matemáticas, entre ellas están cinco de ingenierías (Energías renovables, industrial, agroindustrial, agronómica y ambiental) y tres de educación (Física Matemática, Ciencias Naturales y matemáticas), mientras que en ciencias económicas se trabaja la parte estadística, en donde los estudiantes ponen en práctica el aprender haciendo con el uso de laboratorios, medios tecnológicos que contribuyen significativamente en el aprendizaje de los estudiantes.

Al estar las asignaturas de física y matemática, presente en las carreras universitarias, se vuelve necesario cambiar la forma de facilitar aprendizajes y utilizar la simulación de laboratorios virtuales para lograr que los estudiantes adquieran ese conocimiento científico que les permita insertarse en su campo laboral. El uso de diferentes plataformas, también contribuye a este proceso de aprendizaje, para el desarrollo de clases, de tal forma de hacer prevalecer la interacción entre estudiantes y docente de una manera activa. Lo anterior implica que los docentes nos enfrentamos a un nuevo reto: dar clases de manera virtual o semipresencial (mixto). Como docentes de matemáticas, física o de cualquier otra disciplina, uno de los objetivos a tener presente es tratar de aprovechar los diferentes medios de comunicación y de información, actualizarse y que de esta manera los estudiantes puedan desarrollar habilidades, destrezas, en el contexto de estudio.

Tomando como referencia, que la mayoría de estudiantes son nativos digitales ${ }^{8}$, se vuelve fácil el uso de entornos virtuales a la par de clases presenciales, para dar un aprendizaje con calidad y pertinencia. Claramente, la tecnología por sí sola no es la que produce aprendizajes, esta va acompañada de la metodología y experiencia del docente, la cual es muy importante en estos tiempos de pandemia.

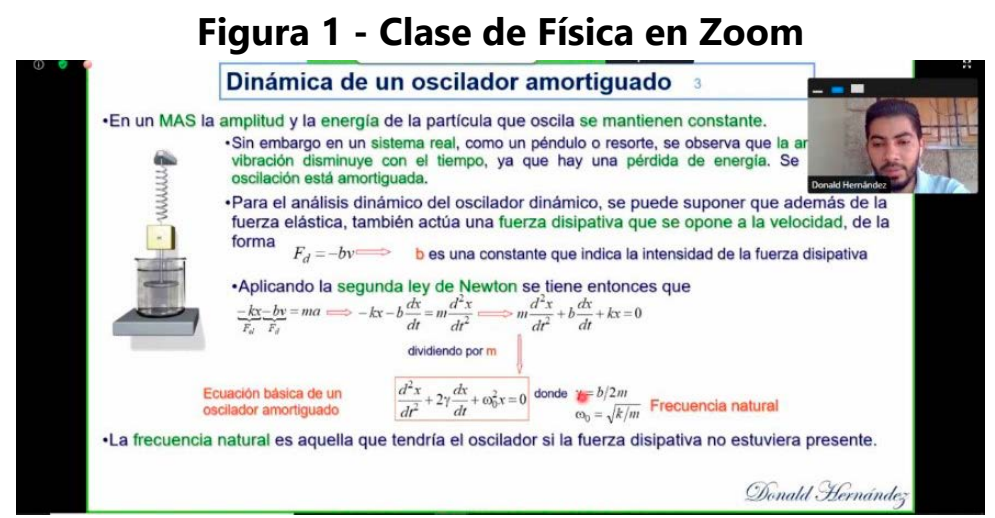

Fuente: Los autores

8 El término "nativo digital" fue acuñado por el autor estadounidense Marc Prensky en 2011 en un ensayo titulado "La muerte del mando y del control"; en él los describía como aquellas personas que habían crecido con la red y el progreso tecnológico y lo que estos implicaban 
En contextos complicados en el proceso de enseñanza aprendizaje, corresponde considerar que los recursos tecnológicos no reemplazarán la labor educativa, pero sí se pueden convertir en una herramienta fundamental para generar un significativo proceso de enseñanza-aprendizaje; dados que el buen uso de estos recursos promoverá un escenario adecuado y servirán de conexión entre docente y estudiantes. Cuando se habla del buen uso de las TIC, principalmente en el área de Física y de matemática entra en juego, un termino clave en tiempos de pandemia como lo es, la planificación y evaluación.

Tal como la resalta Delgado (2020), los educadores deben usar estrategias educativas que se enfoquen en la evaluación formativa para realmente saber qué tan bien están aprendiendo los alumnos y hacer ajustes si es necesario, ser flexibles es fundamental. Según Delgado (2020), existen ocho prácticas docentes de enseñanza de matemáticas equitativas y efectivas ya que sirven para provocar que los estudiantes piensen más. Estas prácticas se enfocan en la evaluación formativa. Esto sirve, por ejemplo, al querer obtener y usar evidencia del pensamiento matemático de los estudiantes y que se sientan valorados. Para implementarlos, se necesita que el docente plantee preguntas con propósito, analice las tareas y trabajos y observe a los alumnos. Estas ocho prácticas efectivas son:

- Establecer objetivos matemáticos para enfocar el aprendizaje

- Implementar tareas que promuevan el razonamiento y la resolución de problemas

- Usar y conectar representaciones matemáticas

- Facilitar un discurso matemático significativo

- Plantear preguntas con propósito

- Desarrollar fluidez procesal a partir de la comprensión conceptual

- Apoyar la lucha productiva en el aprendizaje de las matemáticas

- Obtener y usar evidencia del pensamiento de los estudiantes

Por otra parte, la evaluación en línea requiere que el docente conozca algunas herramientas tecnológicas, pero no es suficiente su dominio, sino también repensar y rediseñar las estrategias evaluativas. Lo anterior hace mucha relación a lo que Avilés (2005) plantea en su artículo, relacionado a la reseña de "Los siete saberes necesarios para la educación del futuro" de Edgar Morín, en tiempos de pandemia, se retoman dos ideas contempladas por Morin, las cuales son:

1. Saber afrontar las incertidumbres

2. Saber enseñar la comprensión

El primer saber (afrontar las incertidumbres) estipula que en innumerables momentos y ante infinidad de situaciones, hemos sentido incertidumbre frente a lo inesperado, y es que precisamente lo incierto suele darle al hecho educativo una perspectiva de inseguridad y vulnerabilidad que desboca el desequilibrio e inestabilidad. Ante esto, Morin cuestiona ¿por qué no enseñar principios de estrategias que permitan afrontar los riesgos?, y es que de alguna manera, el académico antes mencionado, asume que dando respuesta a la pregunta vertida, se puede buscar que los docentes puedan enfrentar lo inesperado y modificar el desarrollo de su actuar formativo con base en las informaciones previamente adquiridas y planeadas; potenciando que el buen docente deba siempre tener en mente varias alternativas de solución a los problemas. 
Enseñar matemática, física o cualquier otra disciplina, en tiempos de pandemia, y de manera virtual, también conlleva a afrontar grandes desafíos, como lo es la equidad educativa, al analizarla desde el punto de vista de aquellos casos de estudiantes vulnerables, que no cuentan con conectividad y aparatos tecnológicos. Por más que no se desee, existirá una desventaja entre aquellos estudiantes de zonas urbanas con familias con posibilidad económica y entre aquellos educandos de la zona rural que no cuentan con recursos y los medios necesarios, para continuar sus estudios de forma virtual.

Por otro lado, ante esta nueva realidad surgida por las consecuencias del COVID-19, la educación nicaragüense se ha visto en la necesidad de ajustarse a un nuevo escenario formativo. Esto ha permitido que los hogares de cada estudiante no solo sea el espacio en donde se aprenden valores, va más allá, se han convertido en los nuevos escenarios de aprendizaje formal y, también, informal.

\section{CONCLUSIONES}

A continuación, se presentan las principales conclusiones de este escrito:

- Es importante mencionar que el inicio de la virtualización de los procesos de enseñanza -aprendizaje no comienza con este problema del COVID-19, sino que, desde antes, pero ahora ha alcanzado mayor realce.

- Incorporar las nuevas tecnologías de la información y comunicación al proceso de enseñanza aprendizaje en el área de física y de matemática es uno de los grandes retos, ya que no basta con estrategias momentáneas; requiere un proceso de transformación desde sus bases.

- Sí los docentes tienen una adecuada formación en metodologías digitales permitirá que éste cuente con herramientas acertadas para acompañar a los estudiantes y familias nicaragüenses en esta nueva realidad virtual.

- La evaluación en línea de la matemática y física requiere que el docente conozca las herramientas tecnológicas, pero no es suficiente su dominio, sino también repensar y rediseñar las estrategias más adecuadas.

- Los estudiantes se han convertido en protagonistas y creadores de su propio itinerario de aprendizaje y con las TIC, se promueve a la acción, a tocar, a conocer y a vivenciar.

- Las universidades están haciendo todo lo posible por garantizar la continuidad educativa y a la vez mantener la calidad científica y humanística, que caracteriza a la UNAN -Managua.

\section{REFERENCIAS}

Avilés Anaya, H. (2005). Reseña de "Los siete saberes necesarios para la educación del futuro" de Edgar Morín. Ra Ximhai, 653-665. Obtenido de http://www.redalyc.org/articulo.oa?id=46110314

Consejo Nacional de Universidades CNU. (17 de Abril de 2020). COMUNICADO OFICIAL 02-2020 DEL CONSEJO NACIONAL DE UNIVERSIDADES. Comunicado de Prensa. Managua, Nicaragua.

Delgado, P. (21 de Julio de 2020). Enseñanza de las matemáticas en la era de COVID - 19. Obtenido de https://observatorio.tec.mx/edu-news/ensenanza-de-las-matematicas-covid19 
Herrera Castrillo, C. J. (2021). Aprendizaje en las asignaturas "Electricidad" y "Termodinámica y Física Estadística" en tiempos de pandemia. Revista Multi-Ensayos, 7(13), 14-25.

Sánchez, J. A. (24 de Marzo de 2020). Virtualidad: la pandemia que cambió la educación superior para siempre. Obtenido de https://www.eltiempo.com: https://www.eltiempo.com/vida/ciencia/ educacion-virtual-la-pandemia-que-cambio-la-educacion-superior-para-siempre-476390 